\title{
Note on a Resolution of Linear Differential Systems
}

By I. S. and E. S. SokolNIKoff, University of Wisconsin.

Communicated by J. M. WhitTaker.

(Received 4th August, 1933. Read 4th November, 1933.)

In a recent paper ${ }^{1} \mathrm{~J}$. M. Whittaker considered the problem of resolving a linear differential system into a product of two or more systems of lower order. This note is a contribution to this problem and furnishes the necessary and sufficient conditions for the resolution of the system into two equivalent systems of lower order of the type considered by Whittaker.

Consider the linear differential system

$$
\begin{gathered}
P y=f(x) \\
\gamma_{i}=\sum_{j=0}^{n-1}\left[a_{i j} y^{(j)}(a)+\beta_{i j} y^{(j)}(b)\right], \quad(i=1,2, \ldots, n),
\end{gathered}
$$

where $P$ is the linear differential operator

$$
P \equiv \sum_{j=0}^{n} p^{j}(x) \frac{d^{j}}{d x^{j}}
$$

It is assumed that the $p_{j}(x)$ are continuous functions in the closed interval $(a, b)$. The $a_{i j}, \beta_{i j}$ and $\gamma_{i}$ are prescribed constants.

It is desired to find the conditions under which the system (I) can be resolved into the two equivalent systems of lower order

$$
\begin{aligned}
Q y & =z(x) \\
\sum_{j=0}^{s-1}\left[u_{i j} y^{(j)}(a)+v_{i j} y^{(j)}(b)\right] & =\nu_{i}, \quad(i=1,2, \ldots, s)
\end{aligned}
$$

and

$$
R z=f(x)
$$

$$
\sum_{j=0}^{m-1} c_{i j} z^{(j)}(a)+d_{i j} z^{(j)}(b)=\delta_{i}, \quad(i=1,2, \ldots, m)
$$

1 J. M. Whittaker, Proceedings of the Edinburgh Mathematical Society (2), 3 (1932), 10-15. 
where

$$
Q \equiv \sum_{j=0}^{s} q_{j}(x) \frac{d^{j}}{d x^{j}}, \quad R \equiv \sum_{j=0}^{m} r_{j}(x) \frac{d^{j}}{d x^{j}},
$$

$n=m+s$, and $P \equiv R Q$.

Assume that there exist $m$ linearly independent combinations of the $\gamma_{i}$ of the form

where

$$
C_{i} Q y(a)+D_{i} Q y(b)=\delta_{i}, \quad(i=1,2, \ldots, m)
$$

$$
C_{i} \equiv \sum_{j=0}^{m-1} c_{i j} \frac{d^{j}}{d x^{j}} \quad \text { and } \quad D_{i} \equiv \sum_{j=0}^{m-1} d_{i j} \frac{d^{j}}{d x^{j}}
$$

and the $c_{i j}$ and $d_{i j}$ are constants. Then there must exist constants $l_{i k}$ such that

(A) $\sum_{k=1}^{n} l_{i k} \gamma_{k} \equiv\left[\sum_{j=0}^{m-1} c_{i j} \frac{d^{j}}{d x^{j}} Q y\right]_{x=a}+\left[\sum_{j=0}^{m-1} d_{i j} \frac{d^{j}}{d x^{j}} Q y\right]_{x=b},(i=1,2, \ldots, m)$.

Moreover, the constants $l_{i k}, c_{i j}$ and $d_{i j}$ must be chosen so that the $m$ relations given in (A) shall be linearly independent.

If the expressions for $\gamma_{k}$ and $Q$ are substituted in (A), the latter can be written ${ }^{1}$ as

$$
\begin{aligned}
\left(\mathrm{A}^{\prime}\right) \quad \sum_{k=1}^{n} l_{i k} \sum_{j=0}^{n-1}\left[a_{k j} y^{(j)}(a)+\beta_{k j} y^{(j)}(b)\right] \equiv c_{i 0} \sum_{k=0}^{s} q_{s-k}(a) y^{(8-k)}(a) \\
+\sum_{j=1}^{m-1} c_{i j} \sum_{k=0}^{s}\left[D q_{s-k}+D y^{(s-k)}\right]_{x=a}^{j}+d_{i 0} \sum_{k=0}^{s} q_{s-k}(b) y^{(8-k)}(b) \\
+\sum_{j=1}^{n-1} d_{i j} \sum_{k=0}^{s}\left[D q_{s-k}+D y^{(8-k)}\right]_{x=b}^{j}, \quad(i=1,2, \ldots, m) .
\end{aligned}
$$

If the terms in $y(a), y^{\prime}(a), \ldots, y^{(n-1)}(a), y(b), y^{\prime}(b), \ldots, y^{(n-1)}(b)$ are collected in each member, each of the $m$ identities of $\left(A^{\prime}\right)$ becomes an identity between two homogeneous linear forms in the $y(a), \ldots, y^{(n-1)}(a), y(b), \ldots, y^{n-1}(b)$. In order to write down

${ }^{1}$ Using Leibnitz's Theorem $D^{n}(u v)=[D u+D v]^{n}$. 
these identities in a compact form, it is convenient to introduce certain symbols which are defined as follows:

(1) $q_{k}^{(l)}(x) \equiv 0$ for $k>n-m=s$, for all values of $l$.

(2) $\left(\begin{array}{l}j \\ 0\end{array}\right) \equiv 1$ for all $j$.

Then it is readily checked that $\left(A^{\prime}\right)$ becomes

$$
\begin{aligned}
& \sum_{k=0}^{n-1} \sum_{j=1}^{n} a_{j k} l_{i j} y^{(k)}(a)+\sum_{k=0}^{n-1} \sum_{j=1}^{n} \beta_{j k} l_{i j} y^{(k)}(b) \\
& \equiv \sum_{k=0}^{n-1}\left[q_{k}(a) c_{i 0}+\sum_{j=1}^{m-1}\left\{\sum_{t=0}^{j}\left(\begin{array}{l}
j \\
t
\end{array}\right) q_{k-t}^{(j-t)}(a)\right\} c_{i j}\right] y^{(k)}(a) \\
& +\sum_{k=0}^{n-1}\left[q_{k}(b) d_{i 0}+\sum_{j=1}^{m-1}\left\{\sum_{t=0}^{j}\left(\begin{array}{l}
j \\
t
\end{array}\right) q_{k-t}^{(j-t)}(b)\right\} d_{i j}\right] y^{(k)}(b),
\end{aligned}
$$

where $i$ takes the values $1,2, \ldots, m$. In order that each of these identities be satisfied, the corresponding coefficients of $y(a), y^{\prime}(a), \ldots, y^{(n-1)}(a), y(b), y^{\prime}(b), \ldots, y^{(n-1)}(b)$ must be equal. Equating coefficients and transposing all terms to the left side gives the following set of $2 n$ equations in the $l_{i j}, c_{i j}$ and $d_{i j}$.

$$
\sum_{j=1}^{n} a_{j k} l_{i j}-q_{k}(a) c_{i 0}-\sum_{j=1}^{m-1}\left\{\sum_{t=0}^{j}\left(\begin{array}{l}
j \\
t
\end{array}\right) q_{k-t}^{(j-t)}(a)\right\} c_{i j}=0
$$

$$
\begin{gathered}
\sum_{j=1}^{n} \beta_{j k} l_{i j}-q_{k}(b) d_{i 0}-\sum_{j=1}^{m-1}\left\{\sum_{t=0}^{j}\left(\begin{array}{l}
j \\
t
\end{array}\right) q_{k-t}^{(j-t)}(b)\right\} d_{i j}=0, \\
(k=0,1,2, \ldots, n-1), \quad(i=1,2, \ldots, m) .
\end{gathered}
$$

From the form of (B) it is clear that the set of equations in $l_{i j}, c_{i j}$, and $d_{i j}$ is the same for all values of $i=1,2, \ldots, m$. It follows that the system (B) must have $m$ linearly independent solutions if there are to be $m$ linearly independent boundary conditions. Therefore, this system of $2 n$ equations in $n+2 m$ unknowns must have a rank $R \leqq n+m$. For, if $R$ is the rank, then there are at most $n+2 m-R$ linearly independent solutions. If $n+2 m-R \geqq m$, then $R \leqq n+m$. 
The matrix $\Delta$ of the system (B) is

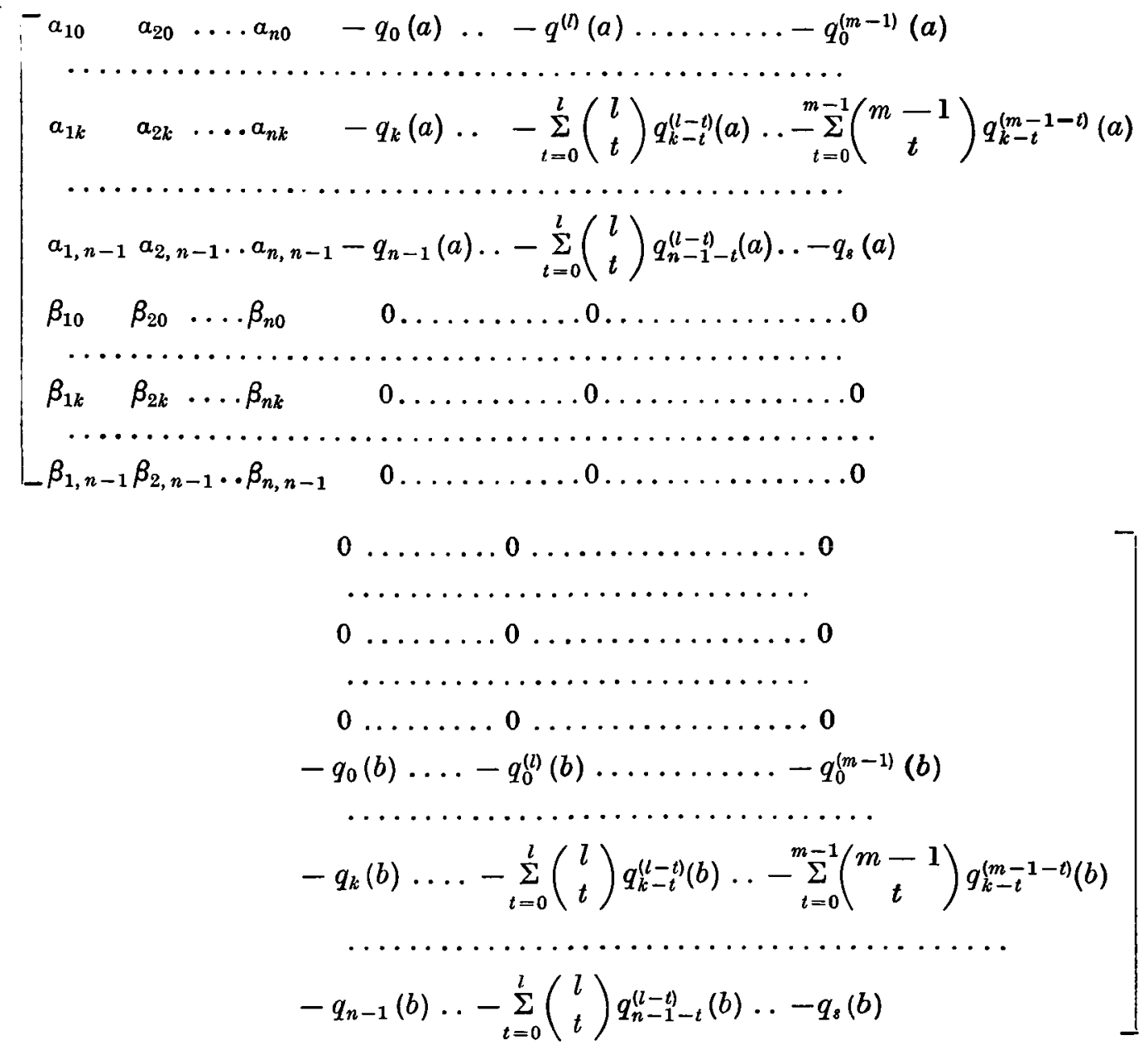

If this matrix has $\operatorname{rank} R \leqq m+n$, then there are at least $m$ linearly independent combinations of the $\gamma_{i}$ which can be expressed in the form

$$
C_{i} z(a)+D_{i} z(b)=\delta_{i}, \quad(i=1,2, \ldots, m) .
$$

These are the boundary conditions for the equation

$$
R z=f(x) \text {. }
$$

Having obtained these $\vec{j} m$ linear combinations, there exist $n-m=s$ other linearly independent combinations of the $\gamma_{i}$ such that the entire set is linearly independent. These $s$ combinations contain, in general, $y(a), y^{\prime}(a), \ldots, y^{(n-1)}(a), y(b), y^{\prime}(b), \ldots, y^{(n-1)}(b)$. 
By successive differentiations of $Q y=z(x)$, the derivatives of order greater than $s-1$ can be eliminated, since $z(x)$ can be regarded as known from the solution of system (III). The resulting $s$ combinations, $\sum_{j=0}^{s-1}\left[u_{i j} y^{(j)}(a)+v_{i j} y^{(j)}(b)\right]=\nu_{i}, \quad(i=1,2, \ldots, s)$, can be taken as the boundary conditions for the equation

$$
Q y=z(x) \text {. }
$$

This discussion may be summarized as:

Theorem

The necessary and sufficient condition for the resolution of the differential system $(I)$ of order $n$ into the two component systems (II) and $(I I I)$ of orders $m$ and $s,(n=m+s)$, is that the matrix $\Delta$ be of rank $R \leqq m+n$.

For the second order case the condition on the rank of the matrix requires that the determinant of $\Delta$ be zero. This can be expressed in a more convenient form as

$$
\left|\begin{array}{ll}
a_{10} & q_{0}(a) \\
a_{11} & q_{1}(a)
\end{array}\right| \cdot\left|\begin{array}{ll}
\beta_{20} & q_{0}(b) \\
\beta_{21} & q_{1}(b)
\end{array}\right|-\left|\begin{array}{c}
a_{20} q_{0}(a) \\
a_{21} q_{1}(a)
\end{array}\right| \cdot\left|\begin{array}{c}
\beta_{10} q_{0}(b) \\
\beta_{11} q_{1}(b)
\end{array}\right|=0
$$

